\title{
Crystalline Silicon Heterojunction Solar Cell With 81.6\% Fill Factor, Deposited by Physical Vapour Deposition Methods Only
}

\author{
Erenn Ore \\ Department of Engineering \\ University of Cambridge \\ Cambridge, UK \\ eo250@cam.ac.uk
}

\author{
Gehan Amaratunga \\ Department of Engineering \\ University of Cambridge \\ Cambridge, UK \\ gaja1@cam.ac.uk
}

\begin{abstract}
It is shown here that silicon ( $\mathrm{Si}$ ) based solar cells can be deposited by physical vapour deposition (PVD) methods only. These cells are called the PVD-Si cells. Their processing eliminates the deposition methods involving high temperature processing steps, and / or expensive, toxic and flammable gases used for processing conventional solar cells with Si absorbers. The PVD-Si cell design investigated has the structure of ITO $\mid$ MoOx | c-Si | LiF | Al. When this cell is deposited on an untextured crystalline (c-Si) wafer, it has a fill factor value of $81.6 \%$ under the standard test conditions (STC). In order to improve the amount of short-circuit current density (Jsc) generated, this cell is also demonstrated on a textured c-Si wafer, achieving Jsc of 35.94 $\mathrm{mA} / \mathrm{cm} 2$ under STC.
\end{abstract}

Keywords-heterojunction, crystalline silicon, physical vapour deposition, solar cell

\section{INTRODUCTION}

Among the single junction solar cell technologies, crystalline silicon (c-Si) heterojunction (SHJ) solar cell with the thin layers of intrinsic $a-S i: H(i-a-S i$ : $H)$, i.e. the HIT solar cell, has the highest power conversion efficiency $(\eta)$ recorded under the standard test conditions (STC) [1]. In this cell design, a $c$-Si wafer is used as the absorber, where all of the other layers are deposited onto the wafer. Here, the core photovoltaic structure is referred to as WAB where a $c-S i$ absorber (A) is sandwiched between a window contact layer (WCL) and a back contact layer (BCL). A solar cell structure is completed by contacting WAB by a different electrode on either side. Typically, WCL is contacted with a transparent front electrode (TFE), and BCL is contacted with a reflective back electrode (RBE). Photons enter the cell from the TFE side, and reach to absorber through WCL.

Reducing recombination at the surface states of the $c$-Si wafer is critical for achieving high $\eta$ [2]. Thus, the WCL and BCL pair need to be able to passivate the wafer surface states. Furthermore, the WCL and BCL pair need to facilitate the separate collection of photogenerated electrons and holes from the absorber. For the SHJ cell, the WCL and BCL pair are made up by thin film silicon or its alloys (TF-Si/A). For the HIT cell, WCL is made up by a $i-a-S i: H$ layer and a $p$-doped TF-Si/A layer such as $p-a-S i: H$, and BCL is made up by a $i-a-S i: H$ layer and an $n$-doped TF-Si/A layer such as $n-a$-Si:H [2].
Here, $i-a-S i: H$ covers both sides of the wafer and provides the required interface passivation between the wafer and the doped TF-Si/A layers [2]. The $p$-doped TF-Si/A layer provides hole selectivity, and the $n$-doped TF-Si/A layer provides electron selectivity [2].

The main challenges to improve SHJ cell's performance and processing cost are associated with $\mathrm{WCL}$ and BCL. For example, because of the high density of tail states, the doped TF$\mathrm{Si} / \mathrm{A}$ layers absorb a significant portion of the incoming photons [2]. However, the charge carriers generated by photon absorption in these layers do not contribute to $\eta$ because of the high charge carrier recombination rates [2].

The electrodes of SHJ cells are processed by physical vapour deposition (PVD) methods, whereas TF-Si/A based WCL and BCL are processed by chemical vapour deposition (CVD) methods [2][3], which area more complex and costly compared to PVD methods. Processing TF-Si/A layers involves expensive, toxic and flammable gases with significant health and safety related concerns [4][5]. Consequently, handling these gases requires strict safety procedures, the use of elaborate containment and sophisticated monitoring systems. These factors increase the cell processing costs. Furthermore, in order to prevent cross-contamination, each TF-Si/A layer requires its own CVD deposition chamber. Employing a multi-chamber CVD system further increases the processing complexity and costs [6].

The purpose for this work is to investigate a SHJ cell design that is deposited by PVD methods only, i.e. the PVD-Si cell. This requires replacing the TF-Si/A based WCL and BCL layers by alternative materials, which can be processed by PVD onto a $c$-Si wafer, and which can passivate the $c$-Si surface states. In addition, the alternative WCL and BCL materials should ideally have wider band gaps than TF-Si/A, so that the parasitic absorption losses are reduced. Furthermore, the alternative WCL and BCL materials should provide selective hole conduction and selective electron conduction respectively.

The transition metal oxides with wide band gaps and high work functions, such as $\mathrm{MoO}_{x}, W O_{x}, V_{2} O_{x}$, [3][7]-[10] are identified as suitable materials for WCL. $L i F \mid A l[8][9][11]$ is identified as a suitable BCL $\mid$ RBE combination. The PVD-Si 
cell design investigated is made up by an ITO TFE, a $M o O_{x}$ WCL, an $n$-doped $c$-Si $(n-c-S i)$ absorber, and a $L i F \mid A l$ based BCL | RBE combination.

\section{Cell Design And Processing}

The reference cell is a HIT cell with an $A l \mathrm{RBE}$. It has the structure of ITO (75nm) $|p-a-S i: H(5 \mathrm{~nm})| i-a-S i: H(7 \mathrm{~nm}) \mid$ $n-c-S i|i-a-S i: H(7 \mathrm{~nm})| n-a-S i: H(9 \mathrm{~nm}) \mid A l(300 \mathrm{~nm})$, where the ITO TFE has a $\mathrm{Ag}$ grid on top, Fig. 1 (a). WAB of this cell is abbreviated by picin, so that the reference cell is also referred to as the picin cell.

When the $p-a-S i$ : $H$ layer of the picin cell is replaced with a $10 \mathrm{~nm}$ thick $\mathrm{MoO}_{x}$ layer, the resulting SHJ cell has the Micin $\mathrm{WAB}$, and this cell is referred to as the Micin cell. In the next iteration, the $n-a-S i: H$ layer of the Micin cell is replaced with a $1.5 \mathrm{~nm}$ thick $L i F$ layer, resulting in a SHJ cell with the MiciL WAB, i.e. the MiciL cell. In the following iteration, the $i-a$ $S i: H$ layers of the $M i c i L$ cell are eliminated, resulting in a PVD$S i$ cell with the $M c L$ WAB, i.e. the $M c L$ cell, Fig. 1(b). In the final iteration, the $n-c$-Si wafer of the $M c L$ cell is textured.
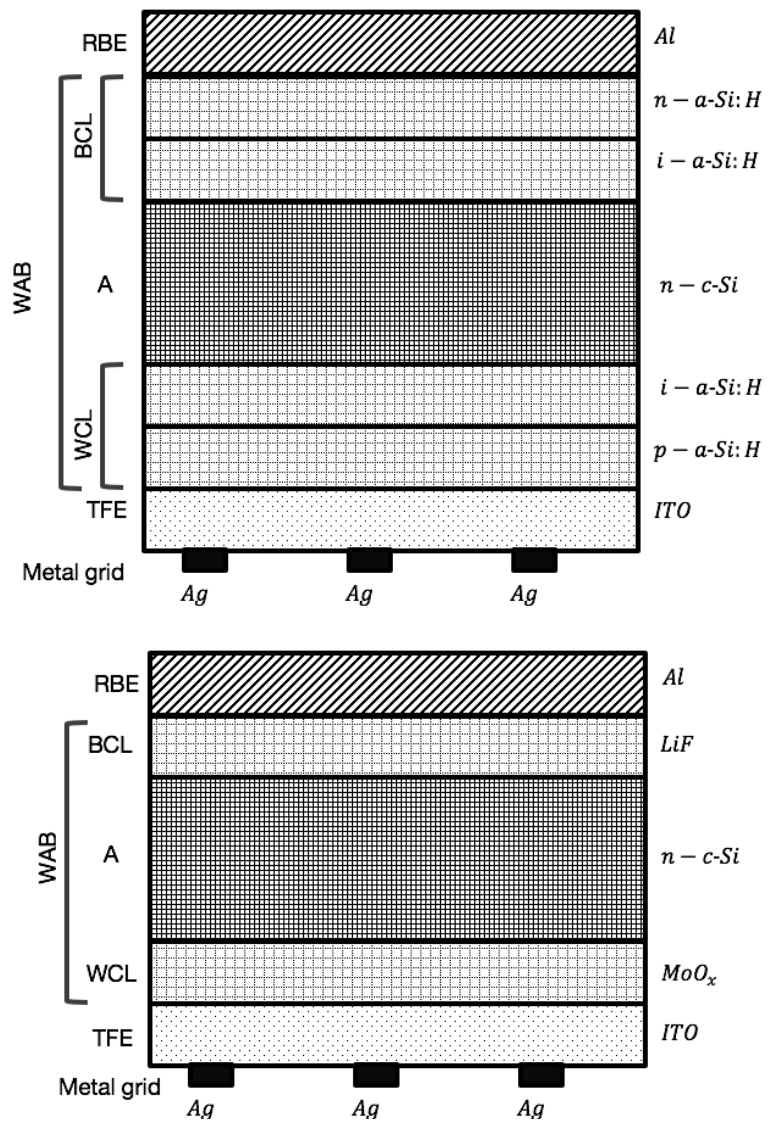

Fig. 1. (a) The reference SHJ cell with the picin WAB, i.e. the picin cell, and (b) the PVD-Si cell with the McL WAB, i.e. the $M c L$ cell.

The wafer used here is phosphorous doped float zone, polished $c$-Si wafer with the thickness of $280 \pm 20 \mu \mathrm{m}$. The textured wafer is prepared by a wet anisotropic etching process. The $p-a-S i: H, i-a-S i: H$ and $n-a-S i: H$ layers are each deposited in a separate CVD chamber of a multi-chamber plasma enhanced CVD system. The non-silicon layers are processed by PVD methods. The solar cell precursors get exposed to air, except between the different $a-S i: H$ deposition steps, and except between the deposition steps of the $L i F$ and $A l$ layers of the MiciL and McL cells. The former is because, during the deposition of the $a-S i: H$ based layers, the cell precursors are transferred between the different CVD chambers without breaking the vacuum. The latter is due to the fact that both $L i F$ and $A l$ layers are deposited sequentially in the same PVD chamber.

The front metal grid defining the cell area of $2 \times 2 \mathrm{~cm}^{2}$ is deposited from a $\mathrm{Ag}$ paste, which is cured for $25 \mathrm{mins}$ at the optimum curing temperature of $200^{\circ} \mathrm{C}$ for the picin cell, and at $130^{\circ} \mathrm{C}$ for the Micin, MiciL and McL cells. The latter is because the efficiencies of the Micin, MiciL and McL cells get reduced when annealed at higher temperatures.

\section{Cell Characterisation}

The series resistance $\left(R_{S}\right)$ and the current density versus voltage $(J V)$ measurements are carried out by a flash type solar simulator under STC. The values for the fill factor $(F F)$ and for the open-circuit voltage $\left(V_{O C}\right)$ are determined from the $J V$ characterisation measurements. The short-circuit current density $\left(U_{S C}\right)$ values quoted are calculated from the external quantum efficiency $\left(\eta_{E Q}\right)$ measurements, which are carried out under the short-circuit condition under STC.

The reflectance and transmittance of the cells are measured by a spectroscopy system at the ambient temperature.

\section{Results And Discussion}

The performance parameters of the $M C L$ cell, which is deposited on a polished $n-c$-Si wafer, are $J_{S C}=32.87 \mathrm{~mA}$ / $\mathrm{cm}^{2}, V_{O C}=0.575 \mathrm{~V}$, and $F F=0.816$. When the $M c L$ cell is deposited on a textured $n-c$-Si wafer, it generates $J_{S C}=$ $35.94 \mathrm{~mA} / \mathrm{cm}^{2}$ under the same conditions.

\section{A. Current Gain Due To Replacing Doped a-Si:H}

The $J_{S C}$ values for the picin and Micin cells are measured to be $31.82 \mathrm{~mA} / \mathrm{cm}^{2}$ and $32.40 \mathrm{~mA} / \mathrm{cm}^{2}$ respectively. Therefore, there is a gain of $0.58 \mathrm{~mA} / \mathrm{cm}^{2}$ in $J_{S C}$, resulting from replacing the $p-a-S i: H$ layer with the $M o O_{x}$ layer. This can be explained by the fact that the photocurrent losses in the $\mathrm{MoO}_{x}$ layer is likely to be less than those in the $p-a-S i: H$ layer, since $\mathrm{MoO}_{x}$ has a wider band gap than $p-a-S i$ : $H$ [12].

When the $n-a-S i$ : $H$ layer of the Micin cell is replaced with the $L i F$ layer, the resulting MiciL cell has $J_{S C}=$ $32.25 \mathrm{~mA} / \mathrm{cm}^{2}$. Thus, there is a reduction of $0.15 \mathrm{~mA} / \mathrm{cm}^{2}$ in $J_{S C}$. This could be due to the $L i F$ layer being only $1.5 \mathrm{~nm}$ thick, which is likely to increase the probability of pinhole formation between RBE and the absorber. Despite this, the MiciL cell generates $0.43 \mathrm{~mA} / \mathrm{cm}^{2}$ more $J_{S C}$ than the picin cell.

\section{B. Current Gain Due To Eliminating $i-a-S i: H$}

In order to investigate the effect of $i-a-S i$ : $H$ on $J_{S C}$, the thickness of the $i-a-S i$ : $H$ layers $(d)$ is reduced incrementally for the MiciL cell. Since $i-a-S i$ : $H$ is commonly used as the absorber for thin film silicon solar cells, some of the photons absorbed in the $i-a-S i$ : $H$ layers is expected to contribute to $J_{S C}$ of the MiciL cell [13]. However, a gradual increase in $J_{S C}$ is 
observed with decreasing $d$, Table I. In fact, eliminating the $i-$ $a-S i$ : $H$ layers from the MiciL cell increases $J_{S C}$ by the amount of $0.62 \mathrm{~mA} / \mathrm{cm}^{2}$. One reason for this behaviour could be resulting from the reduced $R_{S}$, Table I, so that the ohmic losses are reduced and $F F$ is also improved. The other reason could be the reduced reflectance of the $M c L$ cell compared to that of the MiciL cell for the shorter wavelengths, Fig. 2.

TABLE I. THE $J_{S C}, F F$ AND $R_{S}$ VALUES AS A FUNCTION OF THE THICKNESS OF $i-a$-Si: $H$ FOR THE MiciL CELL.

\begin{tabular}{|c|c|c|c|}
\hline$i-a-S i: H(\mathrm{~nm})$ & $J_{s c}\left(\mathrm{~mA} / \mathrm{cm}^{2}\right)$ & $F F$ & $R_{S}\left(\Omega . \mathrm{cm}^{2}\right)$ \\
\hline 8 & 32.12 & 0.744 & 4.65 \\
\hline 6 & 32.34 & 0.756 & 3.98 \\
\hline 4 & 32.65 & 0.775 & 2.35 \\
\hline 0 & 32.87 & 0.816 & 0.77 \\
\hline
\end{tabular}

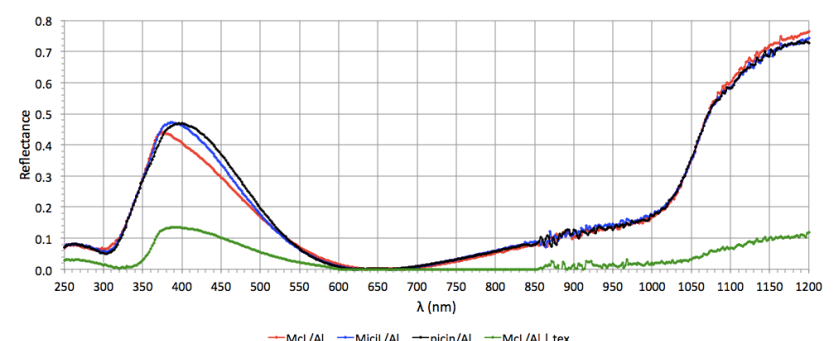

Fig. 2. The reflectance values for the $M c L$, textured $M c L, M i c i L$, and picin cells.

\section{Effects Of Eliminating $i-a-S i: H$ on The Leakage Current And Ohmic Losses}

For the MCL cell and the MiciL cells with different $i-$ $a$-Si: $H$ thicknesses, the $\log _{10}(J)$ versus $V$ curves drawn from the $J V$ measurements taken in dark under ambient conditions are given in Fig. 3.

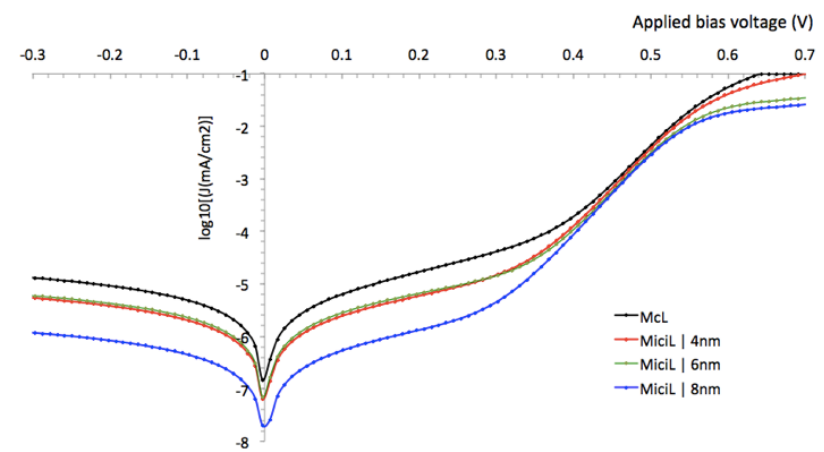

Fig. 3. The $J V$ data taken in dark in ambient conditions for the $M c L$ cell and the MiciL cells with the $i-a-S i$ : $H$ thicknesses of $4 \mathrm{~nm}, 6 \mathrm{~nm}$ and $8 \mathrm{~nm}$.

In the very low forward bias and the reverse bias sections, the leakage current increases as the thickness of the $i-a-S i: H$ layers decreases, indicating that the $i-a-S i$ : $H$ layers covering the $c$-Si wafer surface plays a significant role for blocking the leakage current. In the low reverse bias region, where the curves are almost a linear function of the bias voltage, the recombination saturation current density dominates. The $\log _{10}(J)$ versus $V$ curves in this bias region show that recombination increases with the decreasing $i-a-S i: H$ thickness. This indicates that the $M o O_{x} \mathrm{WCL}$ and the $\mathrm{LiF} \mathrm{BCL}$ without the $i-a-S i$ : $H$ layers do not provide as good $c$-Si wafer surface passivation as the $\mathrm{MoO}_{x} \mathrm{WCL}$ and the $L i F$ BCL with the $i-a-S i$ : $H$ layers.

The diffusion saturation current, which is primarily associated with the junction between the WCL and the absorber, affects the linear segment of the $\log _{10}(J)$ versus $V$ curve at the medium forward bias range. There is a very little difference between the $J V$ curves in this region, thus the $i-a$-Si: $H$ layers do not seem to play a significant role for the back diffusion of charge carriers.

The slope of the curve for the high reverse applied bias region near $V_{O C}$ is correlated with $R_{S}$. Since this slope is decreasing with the decreasing $i-a-S i: H$ thickness, eliminating $i-a-S i$ : $H$ layers reduces $R_{S}$ significantly. As shown in Table I, eliminating the poorly conducting $i-a-S i: H$ layers results in a very low $R_{S}$ of $0.77 \Omega . \mathrm{cm}^{2}$.

In effect, eliminating $i-a-S i$ : $H$ appears to reduce the level of wafer surface passivation and increase the amount of leakage current. However, the associated losses are counterbalanced by the reduced ohmic losses due to the reduced $R_{S}$.

\section{The Effectiveness Of Charge Carrier Separation In The McL cell}

In order to investigate whether the $\mathrm{MoO}_{x} \mathrm{WCL}$ and the $\mathrm{LiF}$ $\mathrm{BCL}$ are able to provide effective charge carrier separation in the $M c L$ cell, the $\eta_{E Q}$ data with no bias and $\eta_{E Q}$ data at the reverse bias of $-1 V$ are compared. According to this comparison, the application of the reverse bias generated extra $J_{S C}$ of $0.07 \mathrm{~mA} / \mathrm{cm}^{2}$, which is insignificant. This implies that an effective charge carrier separation exists in the McL cell.

\section{E. Current Gain Due To Light Trapping}

Photon absorption can be increased by texturing the $c-S i$ wafer surface. The textured $M c L$ cell generates $3.07 \mathrm{~mA} / \mathrm{cm}^{2}$ more $J_{S C}$ than the untextured $M c L$ cell, Fig. 4.

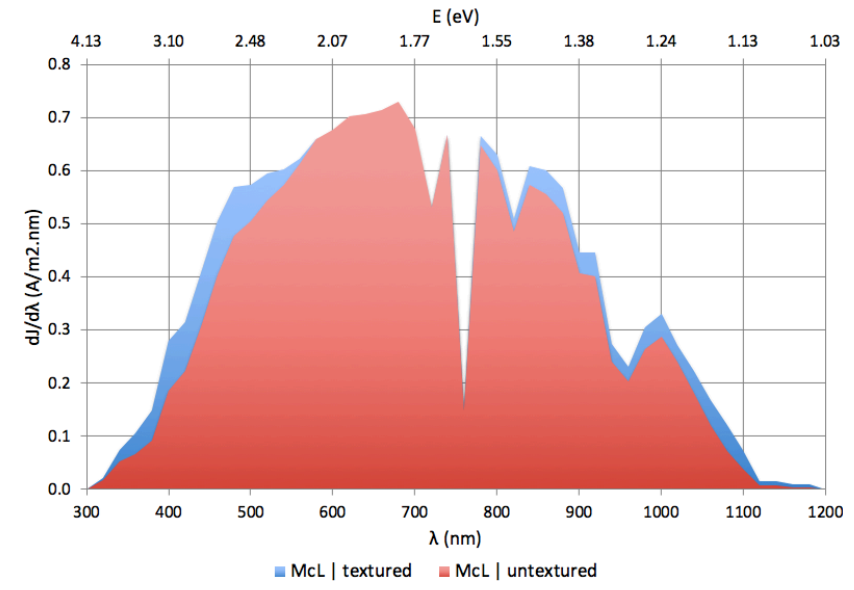

Fig. 4. The spectral photocurrent density $(d J d \lambda)$ distributions for the untextured and textured $M c L$ cells. The area under the $d J d \lambda$ curve gives $J_{S C}$. For the untextured $M c L$ cell, $J_{S C}=32.87 \mathrm{~mA} / \mathrm{cm}^{2}$. When the $M c L$ cell is deposited on a textured $c$-Si wafer, $J_{S C}$ increases by $3.07 \mathrm{~mA} / \mathrm{cm}^{2}$, so that the textured $M c L$ cell generates $J_{S C}=35.94 \mathrm{~mA} / \mathrm{cm}^{2}$. 
The increase in $J_{S C}$ for the textured $M C L$ cell is mostly attributed to the reduced reflectance, Fig. 2, so that more photons can penetrate into the absorber. Furthermore, for photons with the wavelength $(\lambda)$ values beyond $650 \mathrm{~nm}$, scattering at the back of the cell is expected to increase $J_{S C}$ [14]. Thus, some of the additional $J_{S C}$ generated by the textured $M c L$ cell for the longer $\lambda$ is likely to be due to the improved rear reflectance, Fig. 2, resulting from the rough interface between the absorber and $\mathrm{BCL} \mid \mathrm{RBE}$.

\section{F. Losses Due To Imperfect Anti-reflective Coating}

In order to minimise the number of photons reflected from the silver grid deposited onto the ITO TFE, the apex of $\eta_{E Q}$ should be at around $600 \mathrm{~nm}$, where the spectral power density under STC is at its highest value. Since the ITO electrode also acts as the anti-reflective coating, and since the thickness of antireflective layer is depends on the refractive indices of the layers between which it is sandwiched, the apex of $\eta_{E Q}$ can be achieved at around $600 \mathrm{~nm}$ by fine tuning the thickness of the ITO TFE.

For the textured $M c L$ cell and for the untextured picin cell, the $\eta_{E Q}$ peak values occurs at around $600 \mathrm{~nm}$, Fig. 5. Thus, $75 \mathrm{~nm}$ thick ITO TFE appears to have the optimum thickness for these two cells. On the other hand, the highest $\eta_{E Q}$ value for the untextured $M c L$ cell is measured at around $660 \mathrm{~nm}$, indicating that the thickness of the ITO TFE needs to be optimised for this cell.

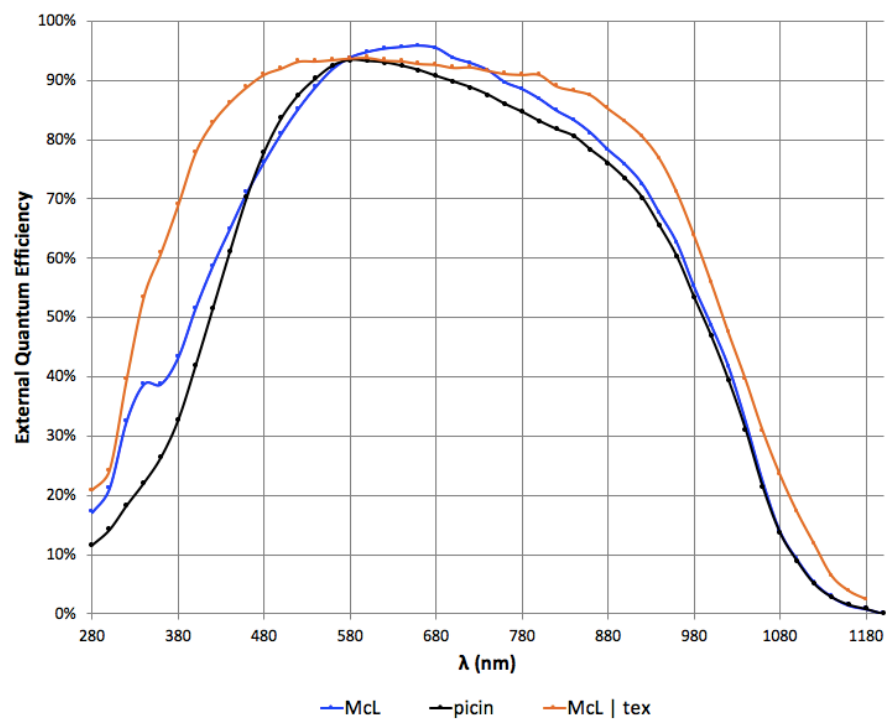

Fig. 5. The external quantum efficiency $\left(\eta_{E Q}\right)$ data for the picin, $M c L$, and textured $M c L$ cells.

\section{G. Potential Improvements}

The main limiting factor for the $M c L$ cell is the relatively low $V_{O C}$, which is likely to be resulting primarily from the air exposure of $\mathrm{MoO}_{x}$. Air exposed $\mathrm{MoO}_{x}$ is observed to have a reduced work function [15], which could be less than the work function of $p-a-S i$ : $H$. This would be detrimental to $V_{O C}$ [2]. Thus, preventing air exposure of the PVD-Si cell precursors after the $M_{0} O_{x}$ deposition step is highly likely to improve $V_{O C}$ significantly.
The textured McL cell processed here was the only attempt to apply surface texturing to the PVD-Si cell concept. Therefore, optimising the screen-printing step of the $\mathrm{Ag}$ grid for the textured $M c L$ cell precursor expected to further improve $V_{O C}$ of the textured $M c L$ cell.

$J_{S C}$ of the untextured MCL cell could be increased by reducing its reflectance, which could be achieved by optimising the thicknesses of the ITO TFE. Furthermore, $J_{S C}$ of both textured and untextured $M C L$ cells could be improved by reducing the probability of pinhole formation between RBE and the absorber. This would require optimising the thicknesses of the LiF BCL.

Note that $\mathrm{MoO}_{x}$ can be replaced with other suitable dopant free materials. For example, compared to $\mathrm{MoO}_{x}$, both $\mathrm{WO}_{x}$ [10] and $V_{2} O_{x}[3]$ are shown to perform better as WCL for SHJ solar cells. Conducting polymers such as PANI [16] can also be used as WCL. Similarly, LiF can be replaced with other suitable dopant free materials, such as $M g O$ [17]. Furthermore, $M g O$ can be combined with $\mathrm{Ag}$ to function as BCL $\mid \mathrm{RBE}$ [17], which could increase $J_{S C}$ due to the better reflectance of $\mathrm{Ag}$ compared to $A l[18]$.

\section{ACKNOWLEDGEMENT}

We are grateful to Prof Miro Zeman of Delft University of Technology, Delft, Netherlands for giving us access to his laboratory for this work.

\section{REFERENCES}

[1] M. A. Green, E. D. Dunlop, D. H. Levi, J. Hohl-Ebinger, M. Yoshita, and A. W. Y. Ho-Baillie, "Solar cell efficiency tables (version 54)", Prog. Photovolt. Res. Appl., vol. 27, pp. 565-575, 2019.

[2] M. Zeman and D. Zhang, "Heterojunction silicon solar cells", in Physics and Technology of Amorphous-Crystalline Heterostructure Silicon Solar Cells, W. G. J. H. M. van Sark and L. K. F. Roca Eds. Berlin \& Heidelberg: Springer-Verlag, 2012, pp. 13-42.

[3] E. Ore, G. Amaratunga, and S. De Wolf, "HIT solar cell with V2Ox window layer", MRS Advances, vol. 2(53), pp. 3147-3156, 2017.

[4] R.S. Crandall, B.P. Nelson, P. D. Moskowitz, and V. M. Fthenakis, "Safety analysis report for the use of hazardous production materials in photovoltaic applications at the National Renewable Energy Laboratory", Report No. NREL/MP-451- 4778A ON: DE92001218,1992.

[5] V. M. Fthenakis and P. D. Moskowitz, "Photovoltaics: environmental, health and safety issues and perspectives", Prog. Photovolt. Res. Appl., vol. 5, pp. 27-38, 2000.

[6] A. Shah, J. Meier, A. Büchel, U. Kroll, J. Steinhauser, F. Meillaud, H. Schade, and D. Domine, "Towards very low-cost mass production of thinfilm silicon photovoltaic (PV) solar modules on glass", Thin Solid Films, vol. 502, pp. 292-299, 2006.

[7] E. Ore, J. Melskens, A. Smets, M. Zeman, and G. Amaratunga, "MoOx hole collection layer for a-Si:H based photovoltaic cells", MRS Advances, vol. 1(14), pp. 977-983, 2016.

[8] E. Ore and G. Amaratunga, "Cylindrical ultra-thin a-Si:H photovoltaic cell with no doped layers", MRS Advances, vol. 2(15), pp. 825-833, 2017.

[9] E. Ore and G. Amaratunga, "Flexible, Dopant Free a-Si:H Solar Cell", in Photovoltaic Specialist Conference (PVSC) [2019 IEEE 46th, IEEE, Chicago, IL, pp. 1135-1138, 2019].

[10] E. Ore and G. Amaratunga, "Crystalline silicon heterojunction solar cells with metal oxide window layers", in Photovoltaic Specialist Conference (PVSC) [2019 IEEE 46th, IEEE, Chicago, IL, pp. 1139-1142, 2019].

[11] E. Ore, J. Melskens, A. Smets, M. Zeman, and G. Amaratunga, "Ultrathin $\mathrm{LiF}$ layer As the electron collector for a-Si:H based photovoltaic cell”, MRS Advances, vol. 2(15), pp. 863-867, 2017. 
[12] M. T. Greiner, L. Chai, M. G. Helander, W. Tang, and Z. Lu, "Transition Metal Oxide Work Functions: The influence of cation oxidation state and oxygen vacancies", Adv. Funct. Mater., vol. 22(21), pp. 4557-4568, 2012.

[13] D. Zhang, I. A. Digdaya, R. Santbergen, R. A. C. M. M. van Swaaij, P. Bronsveld, M. Zeman, J. A. M. van Roosmalen, A. W. Weeber, "Design and fabrication of a SiOx/ITO double-layer anti-reflective coating for heterojunction silicon solar cells", Sol. Energy Mater. Sol. Cells, vol. 117, pp. 132-138, 2013.

[14] M. Zeman, R. A. C. M. M. van Swaaij, J. W. Metselaar, and R. E. I. Schropp, "Optical modeling of a-Si:H solar cells with rough interfaces: effect of back contact and interface roughness", J. Appl. Phys., vol. 88(11), pp. 6436-6443, 2000
[15] S. Subbarayudu, V. Madhavi, and S. Uthanna, "Post-deposition annealing controlled structural and optical properties of RF magnetron sputtered MoO3 films", Adv. Mater. Lett., vol 4(8), pp. 637-642, 2013.

[16] W. Wanga and E. A. Schiff, "Polyaniline on crystalline silicon heterojunction solar cells", Appl. Phys. Lett., vol. 91, p. 133504, 2007.

[17] E. Ore, unpublished.

[18] J. Müller, B. Rech, J. Springer, and M. Vanecek, "TCO and light trapping in silicon thin film solar cells”, Sol. Energy, vol. 77(6), pp. 917-930, 2004. 